\title{
Semi-Markov and hidden semi-Markov models of energy systems
}

\author{
Yuriy E. Obzherin ${ }^{1 *}$ \\ ${ }^{1}$ Sevastopol State University, Higher Mathematics Department, 299053 Sevastopol, Russia
}

\begin{abstract}
The problem of information control systems creation for energy systems and transition to intelligent control and engineering is one of the important problems of reliability and efficiency theory for energy systems. The solution of this problem is possible based on construction of mathematical models concerning different aspects of these systems structure and operation. The possibilities of application of semi-Markov processes with common phase space of states, hidden Markov and semi-Markov models for energy system modeling are considered in the paper.
\end{abstract}

\section{Introduction}

According to the road map of National Technology Initiative "EnergyNet" between the main purposes of this project are:

1."Digitalization" of energy systems, creation of digital networks and digital substations.

2.Creation of information control systems for energy systems and transition to intelligent control and engineering.

The solution of these problems is possible based on mathematical model construction concerning different aspects of energy system structure and operation and development of algorithms and information systems for decision support during design and maintenance of the systems as well as forecast of their states. When constructing adequate models of energy systems it is necessary to use existing possibilities of mathematical apparatus existing.

The possibilities of application of semi-Markov processes with common phase space of states, hidden Markov and semi-Markov models for energy system modeling are considered in the paper.

\section{Semi-Markov processes, hidden semi- Markov models, their application for energy system modelling}

Semi-Markov processes are widely used to construct models and analyse systems of different purposes: technical, production, energy, information, economic, biology etc.

Research in this field is actively carried out in France, the USA, Italy, Great Britain, People Republic of China, India, Russian Federation, Ukraine, Sweden, Japan and some other countries. Under the results of this research there are published several monographs and a large number of papers published. Thus, the leading world publishing houses Elsevier, Springer, World Scientific have recently published the next monographs on this thematic [1 - 7].

In most of these papers semi-Markov models with finite space of states were used. When constructing the models of specific systems it was assumed that most of the random variables characterizing the system have exponential distribution what narrows noticeably the application possibilities of the results obtained.

A large contribution to the development of semiMarkov processes theory and applications was made by works of scientists from the CIS countries V.S. Koroluk, A.F. Turbin, I.A. Ushakov, A.V. Swischuk, V.A. Kashtanov, V.M. Shurenkov. In their works [8 - 13] the algorithms of asymptotic and stationary phase merging are worked out, the models of evolutionary stochastic systems and algorithms of their averaging are built. These papers are actual currently, they provide large opportunities to model systems of different purposes including energy systems.

An effective instrument of system models construction and analysis is the apparatus of theory of semi-Markov processes with common phase space of states [2],[5],[8],[9],[11]. Using of this class of random processes let:

- to get rid of a number of restrictions when model construction, in particulars from the hypothesis on exponential distribution law for random variables characterizing the system;

- to obtain analytic expressions for system's characteristics which can be used for engineering calculations;

- to construct the models for a number of energy systems.

To solve the problem of the dimension of the model one can use the algorithms of asymptotic and stationary phase merging developed by V.S. Koroluk, A.F. Turbin,

Corresponding author: objsev@mail.ru 
A.V. Swischuk [8-11]. This approach opens large opportunities for system modeling.

Since the $80 \mathrm{~s}$ hidden Markov and semi-Markov models have been widely used for system modeling and analysis. In these models there is supposed that observed signal sequence is connected with Markov (semiMarkov) process with non-observable (hidden) states and the task is set to evaluate the structure and characteristics of unobservable process from observations of the signals. A large number of works including recently printed monographs [14-16] is devoted to hidden semi-Markov models.

Hidden semi-Markov models are applied in the next areas: equipment diagnostics and maintenance; reliability of systems, system operation forecasting, network performance evaluation, speech recognition and synthesis, machine translation, pattern recognition, FMRT brain mapping, pathologic events early determination, human activity recognition, protein structure forecasting, financial time series analysis modeling and in some other areas. It is noted in the literature that this approach is used in more than thirty areas.

Semi-Markov processes with common phase space of states and hidden semi-Markov models can be effectively used to construct the models, concerning various aspects of structure and operation of energy systems: reliability, efficiency, control, diagnostics, maintenance and forecasting.

On this basis, the following problems can be solved:

1. To build semi-Markov and hidden semi-Markov models of:

- reliability of energy systems;

- energy systems diagnostics and control;

- energy systems maintenance;

- energy systems forecasting.

2. To find systems technical and economical characteristics suitable for engineering calculations;

3. To solve the problems of energy systems optimum value finding on the basis of objective functions obtained with the aid of the models.

4. To create the software for information systems of energy systems reliability evaluation, control, diagnostic, maintenance and forecasting of states based on the results obtained.

\section{Semi-Markov model of a single-stream line with intermediate storage devices}

We consider a multiphase, single-stream system consisting of serving devices and intermediate storage devices, the connections between which are depicted in Fig.1. Systems of this kind play an important role in the modeling of energy systems [17].

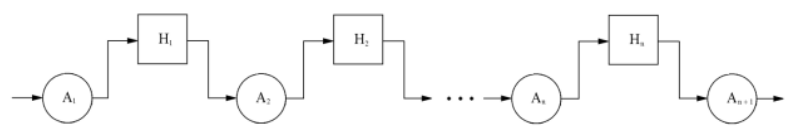

Fig.1. Structural diagram of a multiphase single-flow structure with storage devices.
The following notation is used in the scheme: $A_{i}, i=\overline{1, n+1} \quad-\quad$ service devices; $H_{i}, i=\overline{1, n}-1-$ intermediate storage devices. The model is being constructed under the following assumptions.

1. The possible states of each of the serving devices $A_{i}$ are: operable, recovery and shutdown.

2. Time to failure (renewal time) for the device $A_{i}$ is a random variable $(\mathrm{RV}) \alpha_{i}^{(0)}\left(\alpha_{i}^{(1)}\right)$ with distribution function

$F_{i}^{(0)}(x)\left(F_{i}^{(1)}(x)\right) . \mathrm{RV} \quad \alpha_{i}^{(0)}\left(\alpha_{i}^{(1)}\right)$ are independent and have finite expectations; there are distribution densities $f_{i}^{(0)}(x)\left(f_{i}^{(1)}(x)\right)$ for $F_{i}^{(0)}(x)\left(F_{i}^{(1)}(x)\right)$.

3. The storage devices $H_{i}$ are absolutely reliable devices that have limited capacity $h_{i} \geq 0$ (The storage capacity of the device $H_{i}$ is expressed in units of time that device $A_{i+1}$ will need to fully free this storage device).

4. An operable device $A_{i}$ disconnects, being in operable state, if the storage device $H_{i-1}$ is empty or the storage device $H_{i+1}$ is full .

5.The productivity of device $A_{i}$ is constant and equal to $c_{i}$, herewith $c_{i} \geq c_{i+1}$.

6. The system is in failed state if the output device $A_{n+1}$ does not produce products; the renewal of the device $A_{i}$ is considered as infinite.

To describe the operation of the system let us introduce the next discrete-continuous space of semiMarkov states:

$$
\begin{gathered}
\mathrm{E}=\bar{d} \overline{x z}: i=\overline{1, n+1}, \bar{d}=\left(d_{1}, \ldots, d_{n+1}\right), \\
\bar{x}=\left(x_{1}, \ldots, x_{n+1}\right), \bar{z}=\left(z_{1}, \ldots, z_{n}\right),
\end{gathered}
$$

where $i$ is the number of device $A_{i}$ that failed or was recovered by the last. The element $d_{k}$ of the vector $\bar{d}$ fixes the state of the device: operable $\left(d_{k}=0\right)$, recovery $\left(d_{k}=1\right)$, shut-down $\left(d_{k}=2\right)$. The value of the element $x_{k}$ of the vector $\bar{x}$ is the time lasted from the instance of device $A_{k}$ change of state, it should be noted that $x_{i}=0$. The component $z_{k}$ of the vector $\bar{z}$ defines the time within which the storage device $H_{k}$ can supply the device $A_{k+1}$ with production, $0 \leq z_{k} \leq h_{k}, k=\overline{1, n}$.

To find approximately the reliability characteristics of the system considered let us use the phase merging algorythm [11] which consists in the following.

Let us suppose that stochastic kernel of embedded Markov chain (EMC) $\left\{\xi_{n} ; n \geq 0\right\}$ for the initial system semi-Markov process $\xi(t)$ is close to the stochastic kernel of EMC $\left\{\xi_{n}^{(0)} ; n \geq 0\right\}$ for some supporting system $S_{0}$ with the single stationary distribution $\rho(d x)$.Then the next approximate formulas represented in [11] can be used for approximate finding of the average stationary time to failure $T_{+}$, the average stationary renewal time $T$. and the stationary availability factor $K_{a}$ :

$T_{. .} \approx \frac{\left(\rho, \overline{\mathrm{m}}_{1}\right)}{\left(\rho, P^{(\mathrm{r})} \overline{1}_{0}\right)}, T_{-} \approx \frac{\left(\rho, P^{(\mathrm{r})} \overline{\mathrm{m}}_{0}\right)}{\left(\rho, P^{(\mathrm{r})} \overline{1}_{0}\right)}, K_{\Gamma}=\frac{T_{+}}{T_{+}+T_{-}}$, 


$$
\begin{gathered}
\overline{\mathrm{m}}_{1}(x)=\left\{\begin{array}{l}
m(x), x \in E_{-}, \\
0, x \in E_{-},
\end{array} \quad \overline{\mathrm{m}}_{0}(x)=\left\{\begin{array}{l}
0, x \in E_{-}, \\
m(x), x \in E_{-},
\end{array}\right.\right. \\
\overline{1}_{0}(x)=\left\{\begin{array}{l}
0, x \in E_{+}, \\
1, x \in E_{-},
\end{array} \quad(\rho, f)=\int_{E} f(x) \rho(d x),\right.
\end{gathered}
$$

$\rho(d x)$ is supporting EMC $\left\{\xi_{n}^{(0)} ; n \geq 0\right\}$ stationary distribution; $m(x)$ are the average sojourn times for the states $x \in E$ of the initial system; $P^{(\mathrm{r})}(x, B)$ are the initial system EMC $\left\{\xi_{n} ; n \geq 0\right\}$ transition probabilities; $r$ is the minimum number of steps through which the system can transit to the failure states subset $E$. from the operable states subset $E_{+}$comprised to the ergodic class $E^{0}$.

An important moment of using of the method applied is the choice of the supporting system $S_{0}$. Let us suppose that devices $A_{i}, i=\overline{1, n}$ have fast renewal, i.e. their renewal times $\alpha_{i}^{(1)}$ depends on small positive parameter $\varepsilon$ in such a manner that

$$
\lim _{s \rightarrow 0} E \alpha_{i}^{(1, s)}=0, \quad i=\overline{1, n}
$$

and output device $A_{n+1}$ has fixed time to failure and recovery time. This leads to the fact that recovery system $S_{0}$ will be the system with instant recovery of the devices $A_{i}, i=\overline{1, n}$ and completely filled storage devices $H_{i}$.

Using the formulas (1) one can show that stationary characteristics of the system considered: stationary time to failure $\mathrm{T}_{1}^{\left(h_{1}, \ldots, h_{n}\right)}$ and average stationary renewal time $\mathrm{T}^{\left(h_{1}, \ldots, h_{n}\right)}$ are approximately calculated by the formulas:

$$
\begin{aligned}
& \mathrm{T}_{+}^{\left(h_{1}, \ldots, h_{n}\right)} \approx \prod_{k=1}^{n+1} E \alpha_{k}^{(0)}+\sum_{i=1}^{n} \prod_{k=1}^{n+1} E \alpha_{k}^{(0)} \int_{k \neq i}^{h_{i}} \bar{F}_{i}^{(1, s)}(t) d t / \prod_{k=1}^{n} E \alpha_{k}^{(0)}+ \\
& +\sum_{i=1}^{n} \prod_{k=1,}^{n} E \alpha_{k}^{(0)} \int_{0}^{h_{i}} \bar{F}_{i}^{(1, s)}(t) d t+\sum_{i=1}^{n} \prod_{r=1}^{i-1} E \alpha_{r}^{(0)} \bar{F}_{i}^{(1, s)}\left(\sum_{k=i}^{n} h_{k}\right) \times \\
& \times \prod_{m=i-1}^{n+1} \int_{\substack{m=1 \\
\sum_{l=i}}}^{\infty} \bar{F}_{l}^{(0)}\left(x_{m}\right) d x_{m} \\
& \mathrm{~T}_{-}^{\left(h_{1}, \ldots, h_{n}\right)} \approx\left[E \alpha_{n+1}^{(1)} \prod_{i=1}^{n} E \alpha_{i}^{(0)}+E \alpha_{n-1}^{(1)} \sum_{i=1}^{n} \prod_{\substack{k=1, k \neq i}}^{n} E \alpha_{k}^{(0)} \int_{0}^{h_{i}} \bar{F}_{i}^{(1, s)}(t) d t+\right.
\end{aligned}
$$

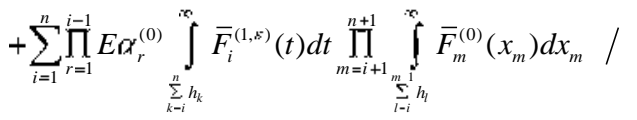

$$
\begin{aligned}
& /\left[\prod_{k=1}^{n} E \alpha_{k}^{(0)}+\sum_{i=1}^{n} \prod_{\substack{k=1, k \neq i}}^{n} E \alpha_{k}^{(0)} \int_{0}^{h_{i}} \bar{F}_{i}^{(1, s)}(t) d t+\right. \\
& \div \sum_{i=1}^{n} \bar{F}_{i}^{(1, k)}\left(\sum_{k=i}^{n} h_{k}\right) \prod_{r=1}^{i-1} E \alpha_{r}^{(0)} \prod_{m=i+1}^{n-1} \int_{\substack{m \\
\sum_{i=i}}}^{\infty} \bar{F}_{m}^{(0)}\left(x_{m}\right) d x_{m} .
\end{aligned}
$$

Knowing the expressions for $\mathrm{T}_{1}^{\left(h_{1}, \ldots, h_{n}\right)}$ and $\mathrm{T}^{\left(h_{1}, \ldots, h_{n}\right)}$, one can find the stationary availability factor $K_{a}^{\left(h_{1}, \ldots, h_{n}\right)}$ by the formula (1).

\begin{tabular}{|c|c|c|c|c|}
\hline \multicolumn{5}{|c|}{$\begin{array}{c}E \alpha_{1}^{(0)}=E \alpha_{2}^{(0)}=E \alpha_{3}^{(0)}=E \alpha_{4}^{(0)}=17 h, \\
E \alpha_{1}^{(1)}=E \alpha_{2}^{(1)}=0,6 h, E \alpha_{3}^{(1)}=E \alpha_{3}^{(1)}=0,8 h, \\
h_{1}=h_{2}=h_{3}=h, \text { processing time }-0,2 h .\end{array}$} \\
\hline $\begin{array}{c}\text { Time } \\
\text { reserve }\end{array}$ & \multicolumn{3}{|c|}{ Analytic modeling } & \multirow{2}{*}{$\begin{array}{c}\begin{array}{c}\text { Simulation } \\
\text { modeling }\end{array} \\
\mathrm{K}_{\Gamma}^{\left(h_{1}, h_{2}, h_{3}\right)}\end{array}$} \\
\hline$h$ & $\begin{array}{c}\mathrm{T}_{-}^{\left(h_{1}, h_{2}, h_{3}\right)}, \\
h \\
h\end{array}$ & $\begin{array}{l}\mathrm{T}_{-}^{\left(h_{1}, h_{2} h_{3}\right)}, \\
h\end{array}$ & $\mathrm{~K}_{\Gamma}^{\left(h_{1}, h_{2}, h_{3}\right)}$ & \\
\hline 0 & 4.250 & 0.700 & 0.859 & 0.879 \\
\hline 0.1 & 6.518 & 0.736 & 0.899 & 0.929 \\
\hline 0.2 & 8.693 & 0.736 & 0.919 & 0.932 \\
\hline 0.3 & 10.507 & 0.780 & 0.931 & 0.923 \\
\hline 0.4 & 11.929 & 0.790 & 0.938 & 0.933 \\
\hline 0.5 & 13.029 & 0.795 & 0.943 & 0.927 \\
\hline 0.6 & 13.885 & 0.797 & 0.946 & 0.937 \\
\hline 0.7 & 14.556 & 0.799 & 0.948 & 0.940 \\
\hline 0.8 & 15.084 & 0.799 & 0.950 & 0.939 \\
\hline 0.9 & 15.501 & 0.800 & 0.951 & 0.935 \\
\hline 1.0 & 15.830 & 0.800 & 0.952 & 0.941 \\
\hline 1.1 & 16.089 & 0.800 & 0.953 & 0.943 \\
\hline 1.2 & 16.292 & 0.800 & 0.953 & 0.945 \\
\hline 1.3 & 16.451 & 0.800 & 0.954 & 0.946 \\
\hline 1.4 & 16.575 & 0.800 & 0.954 & 0.947 \\
\hline 1.5 & 16.671 & 0.800 & 0.954 & 0.945 \\
\hline
\end{tabular}

The Table 1 represents the results of four-phase information system stationary characteristics finding using the formulas (3), (4) and using the simulation modeling. During the modeling it was assumed that all
RV (operation and renewal times for all the devices) are distributed by the exponential law.

Table 1. Modeling results for the four-phase system.

\section{Conclusion}

The paper shows the possibilities of semi-Markov processes with common phase space of states and hidden semi-Markov models application to model construction and operation analysis of energy systems. To solve the dimension problem it is proposed to use the algorithms of asymptotic and stationary phase merging of the systems.

The research was carried out within the state assignment of the Minobrnauki of Russia (№ 1.10513.2018/11.12), with financial support by RFBR (project № 18-01-00392a). 


\section{References}

1. F.Grabski, Semi-Markov Processes: Applications in System Reliability and Maintenance (Elsevier, 2015)

2. Yu. E. Obzherin, E.G. Boyko, Semi-Markov Models: Control of Restorable Systems with Latent Failures (Elsevier, Academic Press, 2015)

3. J. Janssen, R. Manca, Applied Semi-Markov Processes (Springer Science + Business Media, 2006)

4. V.S. Korolyuk, N. Limnios, Stochastic Systems in Merging Phase Space (World Scientific, Imperial Coledge Press, 2005)

5. N. Limnios, G. Oprisan, Semi-Markov Processes and Reliability (Springer Science + Business Media, 2001)

6. J. Janssen, N. Limnios (Eds.), Semi-Markov Models and Applications (Kluwer Academic Publishers, 1999)

7. D. Silvestrov, S. Silvestrov, Nonlinearly Perturbed Semi-Markov Processes (Springer, 2017)

8.V. S. Koroluk, A. F. Turbin, Markovian Restoration Processes in the Problems of System Reliability (Naukova Dumka, Kiev, 1982)

9.V. S. Koroluk, Stochastic System Models Naukova Dumka, Kiev (1989)

10. V.S.Korolyuk, A.V. Swishchuk, Evolutionary Stochastic Systems. Algorithms of Averaging and Di usion Approximation. Mathematics and its Applications (Institute of Mathematics, National Academy of Sciences of Ukraine, Kyiv,2000)

11. A. N. Korlat, V. N. Kuznetsov, M. M. Novikov, A. F. Turbin, Semi-Markov Models of Recoverable Systems and Queuing Systems (Shtiinta, Chisinau, 1991)

12. V. M. Shurenkov, Ergodic Markovian Processes (Nauka, Moscow, 1989)

13. V. A. Kashtanov, A. I. Medvedev, Reliability Theory of the Complex Systems (Theory and Practice) (Fizmatlit, Moscow, 2010)

14. Shun-Zheng Yu, Hidden Semi-Markov Models: Theory, Algorithms and Applications (Elsevier, 2015) 15. V.S. Barbu, N. Limnios, Semi-Markov Chains and Hidden Semi-Markov Models toward Applications: their use in Reliability and DNA Analysis (Springer, 2008)

16. J. Hoek, R.J. Elliott, Introduction to Hidden SemiMarkov Models (Cambridge University Press, 2018) 17.Y. N. Rudenko, I. A. Ushakov, Reliability of power systems, 2nd ed (Novosibirsk, Nauka, 1989) 